\title{
Späte Sehnsucht nach la madre patria: Formen lateinamerikanischer Migration in Spanien
}

\author{
Von Andreas Baumer, Rostock*
}

Spanien hat in den vergangenen Jahrzehnten einen dramatischen Wandel durchlaufen. Aus dem klassischen Auswanderungsland wurde eines der wichtigsten Zielländer für Migranten in Europa. Insbesondere seit Mitte der neunziger Jahre des letzten Jahrhunderts erfuhren die Migrationsprozesse nach Spanien eine einzigartige Dynamisierung, die erst durch die Wirtschaftskrise seit 2008 abgeschwächt wurde. In nur eineinhalb Jahrzehnten hat sich der Anteil von Ausländern an der spanischen Bevölkerung von 1,37 \% (1996) auf 12,08 \% (2009) erhöht - und damit den Durchschnittswert der traditionellen Einwanderungsländer Westeuropas überschritten. ${ }^{1}$

Einen wesentlichen Anteil an diesem Boom haben Migranten aus den Ländern Lateinamerikas. War das Migrationsgeschehen der neunziger Jahre noch von einer Zuwanderung aus Afrika, vornehmlich aus dem Maghreb, geprägt, kam es um die Jahrhundertwende zu einer Verlagerung der wichtigsten Migrationsströme: Jetzt dominierte eine massenhafte Zuwanderung aus Lateinamerika, insbesondere aus den wirtschaftlich verarmten und politisch instabilen Ländern des Andenraums. Bis zur Mitte des neuen Jahrzehnts, zusätzlich befördert durch die Massenmigration von Argentiniern in Reaktion auf die politische und ökonomische Krise, blieb die Dominanz der lateinamerikanischen Migration ungebrochen - in einer Weise, dass vielfach schon eine „Lateinamerikanisierung der Immigration“ ${ }^{2}$ konstatiert wurde. Auch wenn dieser Befund inzwischen angesichts massenhafter Zuwanderung aus den Ländern Mittelosteuropas, insbesondere Rumänien, relativiert werden musste, besteht kein Zweifel daran, dass Lateinamerika neben der EU zur wichtigsten Herkunftsregion von Migranten für Spanien geworden ist - und diese Position aller Voraussicht nach auch in Zukunft behalten wird. Mit einem Anteil von 37,70 \% an der ausländischen Bevöl-

Andreas Baumer, Dr. rer. pol.; wissenschaftlicher Mitarbeiter am Institut für Politik- und Verwaltungswissenschaften, Fakultät für Wirtschafts- und Sozialwissenschaften, Universität Rostock. Email: andreas.baumer@uni-rostock.de

1 Instituto Nacional de Estadística, Padrón municipal 1996-2009. http://www.ine.es/jaxi/ menu.do?type=pcaxis\&path=/t20/e245/\&file=inebase (27.07.2010); eigene Berechnungen. Anteil der Migranten mit ausländischer Staatsangehörigkeit an der spanischen Wohnbevölkerung. Legt man die Zahl aller im Ausland geborenen Einwohner Spaniens zugrunde, kommt man sogar auf einen Anteil von 13,83\%. Vgl. ebd.

2

"Latinoamericanización de la inmigración." Antonio Izquierdo Escribano, Diego López de Lera, Raquel Martínez Buján, Los preferidos del siglo XXI: la inmigración latinoamericana en España, Universidad de la Coruña 2003, S. 1. http://esomi.es/uploads/publicaciones/articulos/2003Izquierdo-Lopez\%20y\%20Matinez_LOS\%20PREFERIDOS.pdf (23.07.2010). Dieses und alle weiteren spanischen Zitate wurden vom Autor ins Deutsche übertragen. Das Original findet sich jeweils in den Fußnoten. 
kerung bilden die Lateinamerikaner gegenwärtig noch vor den EU-Bürgern die größte Zuwanderergruppe in Spanien. ${ }^{3}$

Die lateinamerikanische Migration nach Spanien hat sich im Kontext der allgemeinen Migrationsprozesse entwickelt. Wie Migranten aus anderen Weltregionen auch, wurden die latinos angezogen von einer spezifischen Kombination von Pull-Faktoren, von denen der bedeutsamste die, über einen langen Zeitraum ungebrochene, Nachfrage in bestimmten Sektoren des spanischen Arbeitsmarktes war. Dynamisiert wurde die Migrationsbewegung durch Push-Faktoren in den Herkunftsländern, die ebenfalls in ähnlicher Form in anderen Regionen der Welt anzutreffen waren: Wirtschaftliche Krise, politische Instabilität, Perspektivlosigkeit - „Hunger nach Brot und Horizonten“4 ${ }^{4}$, wie Pilar Ponce für die Migranten aus Ecuador konstatiert hat - sorgten nicht nur in vielen Ländern Lateinamerikas dafür, dass sich ein signifikanter Teil der Bevölkerung in Bewegung setzte. Darüber hinaus wurde die lateinamerikanische Migration nach Spanien aber von einer Reihe von weiteren Faktoren bestimmt, die ihren Ursprung in den vielfältigen Beziehungen zwischen la madre patria und den ehemaligen Kolonien, in der Auswanderungstradition Spaniens, kurz: in der oft bemühten gemeinsamen Geschichte haben.

Die lateinamerikanische Migration nimmt also eine Sonderstellung im allgemeinen Migrationsgeschehen Spaniens ein. Lässt sich diese Sonderstellung auf eine Bevorzugung von Migranten aus Lateinamerika zurückführen? Sind die spanischsprachigen, meist katholischen Zuwanderer aus Lateinamerika tatsächlich „die Bevorzugten des 21. Jahrhunderts"? ${ }^{5}$ Gab und gibt es eine aktive Politik der spanischen Regierungen, die die Zuwanderung aus Lateinamerika gegenüber der aus anderen Regionen fördern soll? Lassen sich hier gar Elemente einer ,,neuen Hispanidad ${ }^{* 6}$ identifizieren? Welche Rolle spielt die - vermeintliche oder tatsächliche - sprachliche, kulturelle und religiöse Nähe der lateinamerikanischen Migration zur Aufnahmegesellschaft in den unterschiedlichen Integrationsdiskursen in Spanien?

Um die genannten Fragen im Kontext der gegenwärtigen lateinamerikanischen Migration in Spanien diskutieren zu können, sollen zunächst die Migrationsprozesse nach Spanien und die Entwicklung der Zuwanderungspolitik der letzten zwei Jahrzehnte skizziert werden. Daran anschließend, werden die einzelnen Phasen der Migration aus Lateinamerika umrissen. Im nächsten Teil werden die gegenwärtigen Formen der lateinamerikanischen Migration in Spanien dargestellt. Am Schluss steht die Analyse der rechtlichen, politischen

Instituto Nacional de Estadística, (Fn. 1).

"Hambre de pan y horizontes." Pilar Ponce, La Inmigración Ecuatoriana en España: Nuevas vidas, nuevos problemas, in: Ponce Leiva, Javier (Hrsg.), Migrantes. Problemas y Ayudas, Quito 2005, S. 97. "Los preferidos del siglo XXI." Izquierdo Escribano et al., (Fn. 2), S. 1.

6 "Nueva hispanidad." Ricard Zapata-Barrero, Gestión de la multiculturalidad en España: un análisis contextual para definir un enfoque europeo de la inmigración. I Seminario Inmigración y Europa. España en la construcción de una política europea de inmigración, Barcelona 2005, S. 87. 
und gesellschaftlichen Rahmenbedingungen, unter denen sich dieser unbestreitbare Boom der Zuwanderung aus Lateinamerika innerhalb der letzten zehn Jahre entfalten konnte.

\section{A. Einwanderungsland Spanien: Entwicklung und Charakteristika der Zuwanderung}

Spanien war lange Zeit eines der klassischen europäischen Auswanderungsländer. In den Jahren 1882 bis 1990 emigrierten insgesamt über sieben Millionen Spanier. Diese Emigration vollzog sich in mehreren Phasen und war anfangs von der kolonialen Vergangenheit Spaniens bestimmt. In einer ersten Welle im letzten Drittel des 19. Jahrhunderts und dann noch einmal in den zwanziger Jahren des 20. Jahrhunderts wanderten Millionen Spanier in die ehemaligen Kolonien in Lateinamerika und in der Karibik aus. Waren diese Emigrationswellen in erster Linie wirtschaftlich bedingt, erlebte Spanien nach dem Bürgerkrieg (1936-1939) eine Massenauswanderung aus politischen Motiven. Zeitweise flohen bis zu einer halben Million Anhänger der spanischen Republik vor dem Terror der Sieger unter General Francisco Franco. Etwa 200.000 dieser Flüchtlinge blieben dauerhaft im Exil. Sie ließen sich größtenteils in Frankreich, aber auch in einigen lateinamerikanischen Staaten, etwa Mexiko oder Argentinien, und in geringerem Maße auch in der Sowjetunion nieder. ${ }^{7}$

Eine vierte Emigrationswelle setzte Mitte der fünfziger Jahre ein. Ab diesem Zeitpunkt wanderten spanische Arbeitskräfte in die Industrieländer Nordwesteuropas aus. Hauptmotiv dieser Migration war die bessere wirtschaftliche Situation in den Zielländern. Für viele spanische Arbeitsemigranten dürfte jedoch auch die Flucht vor den repressiven politischen und sozialen Verhältnissen der Franco-Diktatur eine wichtige Rolle gespielt haben. In dieser Phase verließen rund drei Millionen Spanier ihre Heimat. Mit dem Ende der Anwerbephase in den meisten westeuropäischen Staaten 1973, dem spanischen Demokratisierungsprozess ab 1975 und der ab den achtziger Jahren einsetzenden wirtschaftlichen Konsolidierung Spaniens fand diese letzte Auswanderungswelle ihr Ende. Trotz verstärkter Rückwanderung wies die spanische Migrationsbilanz noch lange Zeit einen negativen Saldo auf. Rund zwei Millionen Spanier leben nach wie vor im Ausland.

In den vergangenen Jahrzehnten wurde Spanien zu einem Zielland für Migration. Auch die Zuwanderung nach Spanien lässt sich in mehrere Phasen unterteilen. Mitte der siebziger Jahre des letzten Jahrhunderts kamen neben sonnenhungrigen Pensionären aus Nordwesteuropa vor allem Lateinamerikaner, die vor den Militärdiktaturen in ihren Heimatländern flüchteten. In den achtziger Jahren folgten zunehmend Arbeitsmigranten aus Lateinamerika. Die rasante Wirtschaftsentwicklung führte zu einer erhöhten Nachfrage etwa nach Haushaltspersonal, so dass Spanien als Einwanderungsland gerade für Frauen aus den ehemaligen Kolonien attraktiv wurde. Diese Migrationsbewegungen wurden dadurch begünstigt, dass bis 1985 Einwanderer aus Lateinamerika, den Philippinen und Äquatorialguinea in Spanien keine Arbeits- und Niederlassungserlaubnis benötigten. Im Zuge des spanischen 
EG-Beitritts musste diese Bevorzugung von Zuwanderern aus den ehemaligen Kolonien eingeschränkt werden - genauso wie die Zuwanderungsbestimmungen insgesamt auf Druck der Europäischen Gemeinschaft sukzessive verschärft wurden. ${ }^{8}$

Trotzdem setzte zu Beginn der neunziger Jahre eine massive Zuwanderung von Migranten, vornehmlich aus den Maghrebstaaten, aber auch aus Osteuropa und Asien, ein und verwandelte Spanien innerhalb von wenigen Jahren in ein Einwanderungsland. Dabei blieb die Zuwanderung in absoluten Zahlen lange Zeit vergleichsweise gering. 1996 lebten 542.314 Ausländer in Spanien, was einem Anteil von $1,37 \%{ }^{9}$ an der Bevölkerung entsprach. 2000 waren es schon 923.879 (2,28 \%) Ein regelrechter Boom entwickelte sich in den Jahren nach der Jahrtausendwende: Bis zum Jahr 2003 hatte sich die Zahl der Ausländer mit 2.664.168 fast verdreifacht (6,24\%). 2009 lebten 5.648.671 Menschen nichtspanischer Nationalität in dem iberischen Land. Das entspricht einem Bevölkerungsanteil von rund $12,08 \%$. Innerhalb weniger Jahre hat Spanien damit eine Entwicklung nachvollzogen, die sich in den übrigen Einwanderungsländern Westeuropas über mehrere Dekaden erstreckt hatte.

Neben dieser enormen Dynamik zeichnet sich die Migration in Spanien durch eine Reihe von weiteren Merkmalen aus, die sie von vergleichbaren Prozessen in anderen europäischen Ländern unterscheiden:

- Hoher Anteil von irregulärer Migration: Es gehört zu den Besonderheiten des spanischen Migrationsgeschehens, dass ein großer Teil der Migranten irregulär ins Land gelangte. Der enormen Nachfrage nach billigen Arbeitskräften in Wirtschafszweigen wie der Landwirtschaft, dem Baugewerbe oder dem Dienstleistungsbereich standen immer restriktivere Einreisbestimmungen gegenüber - mit der Folge, dass die Zahl der irregulären Migranten seit Anfang der neunziger Jahre sprunghaft zunahm. Dieses Phänomen dauerte zumindest bis zur Wirtschaftskrise an. Migranten aus Lateinamerika, insbesondere aus Ecuador und Bolivien, reisen in der Regel legal ein und bleiben dann als overstayer irregulär im Land. Für Migranten aus dem Maghreb und den afrikanischen Staaten südlich der Sahara bleibt meist nur die irreguläre Einreise: Tausende von ihnen warten vor den Grenzzäunen Ceutas und Melillas, den spanischen Exklaven in Marokko, auf eine Möglichkeit, auf spanisches Territorium zu gelangen. Eine weitaus größere Zahl geht das lebensgefährliche Risiko ein, auf dem Seeweg Spaniens Küsten zu erreichen. Nach Angaben von Nichtregierungsorganisationen verloren zwischen 1988 und 2000 fast 6.000 Menschen in den Gewässern zwischen Marokko und Spanien ihr Leben. ${ }^{10}$ Als Reaktion auf die immer effizientere Kontrolle der Straße von Gibraltar und der spanischen Festlandküste versuchten in den letzten Jahren

Andreas Baumer, Chancen und Risiken der spanischen Migrationspolitik, Berlin Risk Brief No. 2. (2008), S. 7-12.

Diese und alle weiteren Angaben: Instituto Nacional de Estadística, (Fn. 1); eigene Berechnungen.

10

Andreas Baumer, Spanien. in: Wolfgang Gieler / Dietmar Fricke (Hrsg.), Handbuch europäischer Migrationspolitiken, Münster 2004, S. 179. 
immer mehr Migranten, per Boot von der westafrikanischen Küste über den offenen Atlantik auf die kanarischen Inseln zu gelangen - eine nochmals gefährlichere Route. Trotzdem stieg die Zahl derer, die dieses hohe Risiko eingehen, lange Zeit beständig. Wurden im Jahr 2000 nach offiziellen Angaben insgesamt 15.195 Migranten bei der illegalen Einreise an Spaniens Küsten festgenommen, waren es 2006 bereits 39.180. Im Jahr 2007 sank die Zahl erstmals wieder auf 18.057. ${ }^{11}$ Seit Anfang der neunziger Jahre nahm die Zahl der irregulären Migranten beständig zu und explodierte förmlich um die Jahrtausendewende. 2003 war rund die Hälfte der Migranten in Spanien ohne regulären Aufenthaltsstatus. 2009 hat sich der Anteil der Irregulären zwar auf ein gutes Fünftel reduziert - umfasst aber noch immer mehr als eine Million Menschen. Trotz einer massenhaften Regularisierung 2005 und einer verschärften Abschiebepolitik bleibt das Phänomen der irregulären Migration also eine zentrale Herausforderung der spanischen Migrationspolitik. ${ }^{12}$

- Phasen ethnischer Dominanz: Die Zuwanderung nach Spanien war in ihren verschiedenen Phasen meist von einer bestimmten Migrantengruppe bestimmt. So gelten die neunziger Jahre als das Jahrzehnt der marokkanischen Migration, wenngleich in diesem Zeitraum auch Migranten aus anderen Regionen nach Spanien kamen. Tatsächlich bildeten aber Marokkaner lange Zeit die mit Abstand größte nationale Gruppe unter der zugewanderten Bevölkerung, einen Rang, den sie erstmals 2008 an die Migranten rumänischer Herkunft abgaben. ${ }^{13}$ Um die Jahrtausendwende setzte der Boom der Zuwanderung aus Lateinamerika ein, in den letzten Jahren schließlich gewann die Migration aus den Ländern Mittelosteuropas an Bedeutung. Betrachtet man Anteil der unterschiedlichen Herkunftsregionen an der heutigen ausländischen Bevölkerung Spaniens, so wird deutlich, dass der Maghreb und Afrika südlich der Sahara ihre frühere Bedeutung zugunsten Lateinamerikas und Mittelosteuropas eingebüßt haben.

- Starke geographische Konzentration: Ein weiteres Merkmal der Zuwanderung nach Spanien ist die Konzentration der Migranten auf wenige Autonome Gemeinschaften. Diese Konzentration folgt den spezifischen Nachfragestrukturen auf dem Arbeitsmarkt und ist daher bei den verschiedenen Migrantengruppen unterschiedlich ausgeprägt. Während beispielsweise marokkanische Migranten oftmals in der arbeitsintensiven Landwirtschaft der südöstlichen Regionen beschäftigt sind, führt etwa die Dominanz von Migrantinnen aus Lateinamerika im Bereich der häuslichen Dienstleistungen zu einer starken Präsenz dieser Gruppe in den Metropolen. Insgesamt lässt sich jedoch festhalten, dass sich der Großteil der Migranten in den Großräumen von Madrid und Barcelona, in den Zentren des Massentourismus sowie in den Regionen mit arbeitsintensiver Landwirtschaft sammelt. ${ }^{14}$

11

12

13

14

El País, 13.03.2008.

Vgl. Baumer, (Fn. 8); Instituto Nacional de Estadística, (Fn. 1).

Instituto Nacional de Estadística, (Fn. 1).

Vgl. Joaquín Recaño Valverde, Andreu Domingo i Valls, Evolución de la distribución territorial y la movilidad geográfica de la población extranjera en España, in: Eliseo Aja / Joaquín Arango 


\section{B. Entwicklung der spanischen Migrationspolitik}

Die spanischen Regierungen brauchten lange Zeit, um auf das Phänomen der massenhaften Zuwanderung mit der Formulierung einer eigenständigen Migrationspolitik zu reagieren. Als klassisches Auswanderungsland mit einer im hohen Maße vom Tourismus abhängigen Wirtschaftsstruktur hatte Spanien lange Zeit Interesse an möglichst unbürokratischen Einreisebestimmungen. Das änderte sich mit dem Beitritt des Landes zur Europäischen Gemeinschaft 1986 und zum Schengener Abkommen 1991. Die Zuwanderungs- und Ausländerpolitik Spaniens wurde fortan bestimmt durch seine politische und geographische Position an der Südflanke der Europäischen Gemeinschaft. Die Voraussetzung für die Teilnahme am Schengener Abkommen war die Übernahme des zuvor von den Kernsstaaten erarbeiteten acquis. Spanien führte 1990 die Visumspflicht für Nicht-EU-Bürger ein. Davon ausgenommen blieben nur Staatsangehörige der Länder, die aus den ehemaligen Kolonien in Lateinamerika hervorgegangen waren. Ab 2001 wurden auch diese Sonderregelungen sukzessive aufgehoben.

Neben der Anpassung (und damit Verschärfung) der einreise- und aufenthaltsrechtlichen Bestimmungen an die entsprechenden Standards der übrigen Schengen-Staaten begann Spanien damit, die Sicherung seiner Grenzen gegen irreguläre Einwanderung voranzutreiben. Ceuta und Melilla wurden mit Mauern, Stacheldrahtzäunen und Überwachungskameras zu regelrechten Festungen ausgebaut. Außerdem wurde ein radar- und infrarotgestütztes Informationssystem aufgebaut, mit dem die spanischen Küstengewässer überwacht und so Boote mit irregulären Einwanderern schon vor der Landung aufgebracht werden sollen. In den letzten Jahren hat die spanische Regierung den Kampf gegen die irreguläre Migration weiter verstärkt. Neben einer verstärkten Sicherung der Seegrenzen - zeitweise auch mit Unterstützung der europäischen Grenzagentur Frontex - wurden insbesondere Rücknahmeabkommen mit einer Reihe von west- und zentralafrikanischen Transit- und Entsendeländern geschlossen und die Abschiebepolitik drastisch verschärft. ${ }^{16}$

Erst unter dem Eindruck der massiven Zuwanderung wurde Ende 1999 ein neues Ausländergesetz (ley de extranjería) beschlossen. Dieses Gesetz war nach einer langen Debatte mit den Stimmen der linken und nationalistischen Oppositionsparteien gegen die Stimmen der regierenden konservativen PP (Partido Popular - Volkspartei) verabschiedet worden. Es trat an die Stelle des alten Ausländergesetzes von 1985 und galt als das fortschrittlichste

(Hrsg.), Veinte años de inmigración en España. Perspectivas Jurídica y sociológica [1985-2004], Barcelona 2006, S. 303-338.

Axel Kreienbrink, Einwanderungsland Spanien. Migrationspolitik zwischen Europäisierung und nationalen Interessen, Frankfurt/Main, London 2004, S. 192-205.

16

Ana María López Sala, El control de los flujos migratorios y la gestión política de las fronteras en España, in: Ricard Zapata-Barrero (Hrsg.), Políticas y gobernabilidad de la inmigración en España, Barcelona 2009, S. 31-49. 
eines Mitgliedsstaates der Europäischen Union. Neben Erleichterungen für reguläre Zuwanderer beinhaltete es auch einige Rechte für irreguläre Migranten wie etwa Zugang zur Gesundheitsversorgung und zur öffentlichen Schuldbildung sowie Vereinigungs-, Streik- und Demonstrationsrechte. ${ }^{17}$ In der Beratungsphase hatte die PP das Gesetzesvorhaben noch unterstützt - ihr Rückzug und die Verabschiedung des Gesetzes gegen die Stimmen der Regierung markierten das Ende des Parteienkonsenses in der spanischen Zuwanderungspolitik.

Nach Wahlen im März 2000 verfügte die konservative Regierung von José María Aznar erstmals über eine absolute Mehrheit im Parlament und kündigte die Verschärfung des gerade erst in Kraft getretenen Gesetzes an. Durch die Rücknahme verschiedener Rechte für Irreguläre sollte die angebliche „Sogwirkung“ des Gesetzes beseitigt werden. Die Reform der ley de extranjería führte zu einer breiten Debatte in der spanischen Öffentlichkeit, an der sich Parteien, Gewerkschaften und verschiedene NGOs, darunter auch Selbstorganisationen von Migranten, beteiligten. Dazu kamen zahlreiche Protestaktionen wie Kirchenbesetzungen durch irreguläre, von Abschiebung bedrohte Migranten. Unter diesem hohen öffentlichen Druck musste sich die Regierung kompromissbereit zeigen. Zwar wurde die Reform des Ausländergesetzes mit vielen umstrittenen Punkten verabschiedet. So sind im reformierten Gesetzeswerk Rechte wie Vereinigungs-, Streik- und Demonstrationsrechte nun an den regulären Aufenthaltsstatus gebunden. Der Anspruch der Irregulären auf Gesundheitsversorgung und Schulbildung blieb - im Unterschied zu fast allen anderen europäischen Ländern - jedoch erhalten. Voraussetzung für die Inanspruchnahme dieser öffentlichen Leistungen ist die Einschreibung in das kommunale Melderegister (padrón municipal). Dies ist auch für Irreguläre gefahrlos möglich; ein Abgleich dieser Daten mit den normalen Ausländerregistern findet nicht statt. ${ }^{18}$

Der Wahlsieg der sozialistischen PSOE (Partido Socialista Obrero Español - Spanische Sozialistische Arbeiterpartei) von José Luis Rodríguez Zapatero im Jahr 2004 fiel mit dem Höhepunkt des beispiellosen Migrationsbooms nach Spanien zusammen. Die sozialistische Regierung unternahm den Versuch, mittels einer umfassenden Neuformulierung der Migrationspolitik auf diese Situation zu reagieren. Der Politikbereich Migration wurde vom Innenministerium ins Ministerium für Arbeit und soziale Angelegenheiten verlagert, was eine Neubewertung symbolisierte: nicht mehr sicherheitspolitische und polizeirechtliche Aspekte der Migration, sondern Fragen der Integration in die Sozialsysteme und in den

Vgl. Liliana Suárez-Navaz, Raquel Macià Pareja, Ángela Moreno García, El Estado y las luchas de los sin papeles en España: Una extensión de la ciudadanía?, in: Dies. (Hrsg.), Las luchas de los sin papeles y la extensión de la ciudadanía. Perspectivas críticas desde Europa y Estados Unidos, Madrid 2007, S. 185-213.

Dieses Verfahren hat für Politik und Wissenschaft einen interessanten Nebeneffekt: Aus der Differenz zwischen den vergebenen Aufenthaltsbewilligungen und den im padrón registrierten Ausländern lassen sich relativ genaue Rückschlüsse auf die Zahl der Irregulären ziehen. Vgl. $M^{a}$. Isabel Carvajal Gómez, Evolución de las cifras de extranjeros con tarjeta o autorización de residencia en vigor (1992-2004), in: Aja / Arango, (Fn. 14), S. 87. Vgl. auch Fn. 34. 
Arbeitsmarkt sollten im Vordergrund stehen. ${ }^{19}$ Das von der Vorgängerregierung verschärfte Ausländergesetz wurde zwar nicht reformiert, jedoch in seinen Ausführungsbestimmungen liberalisiert.

Schwerpunkt der ersten zwei Regierungsjahre Zapateros war die Auseinandersetzung mit der irregulären Migration. Schon zuvor hatte es in Spanien immer wieder Legalisierungsverfahren für so genannte sin papeles („Papierlose“) gegeben; zuletzt erhielten 2000 und 2001 insgesamt rund 460.000 Irreguläre eine Aufenthaltserlaubnis. Im Frühjahr 2005 fand nun die bislang letzte und umfangreichste Regularisierung statt. Rund 577.000 irreguläre Migranten bekamen Papiere. Neu an diesem Prozess war, dass die Arbeitgeber den Antrag auf Regularisierung zu stellen hatten - und damit ihre bislang illegal Beschäftigten bei der Sozialversicherung anmelden mussten. Dieses Verfahren war ein Schlag gegen die Schattenwirtschaft und brachte Geld in die Sozialkassen. NGOs bemängelten allerdings, dass auf diese Weise die Abhängigkeit der Migranten von ihrem Arbeitgeber noch verstärkt werden würde.

Neben der Regularisierung eines großen Teils der schon eingewanderten sin papeles bildete die Abwehr neuer Irregulärer einen weiteren Schwerpunkt der sozialistischen Migrationspolitik. Wie schon die konservative Vorgängerregierung schlossen die Sozialisten weitere bilaterale Rücknahmeabkommen mit Herkunfts- und Transitländern, um die möglichst rasche Rückführung irregulärer Migranten zu ermöglichen. 2007 wurden über $92 \%$ der bei der Einreise aufgegriffenen Irregulären abgeschoben. ${ }^{20}$

Offen bleibt die Frage, wie die sozialistische Regierung künftig mit dem Phänomen der irregulären Migration umgehen wird. Eine erneute Regularisierungskampagne wurde zwar ausgeschlossen, im Gesetz sind jedoch weiterhin individuelle Regularisierungen aufgrund von „Verwurzelung“ (arraigo) in der Gesellschaft vorgesehen. Auch der erfolgreiche Widerstand der spanischen Regierung gegen das Vorhaben, Regularisierungen im 2008 beschlossenen EU-Einwanderungspakt generell auszuschließen, deutet daraufhin, dass sich Zapatero eine solche Möglichkeit zumindest offen lassen will - was angesichts der nach wie vor hohen Zahl von Irregulären auch nachvollziehbar scheint.

Im Kampf gegen die irreguläre Migration setzte die sozialistische Regierung auch auf die Schaffung von Möglichkeiten der regulären Zuwanderung. Die spezifische Nachfragestruktur des spanischen Arbeitsmarktes nach ausländischen Arbeitskräften - also der wichtigste Pull-Faktor für die irreguläre Zuwanderung - sollte in verschiedenen Politiken zur Förderung von regulärer Migration abgebildet werden. Dazu gehören bilaterale Abkommen über temporäre Migration mit einer Reihe von Entsendeländern, Regelungen für Saisonarbeit, sowie eine mit Unternehmerverbänden und Gewerkschaften abgestimmte Liste von schwer zu besetzenden Stellen, für die gezielt ausländische Arbeitskräfte angeworben

Gemma Pinyol i Jiménez, La política de inmigración española como un nuevo instrumento de acción exterior, in: Esther Barbé (Hrsg.), España en Europa 2004-2008, Monografías del Observatorio de Política Exterior Europa, núm. 4, Barcelona 2008, S. 1.

El País, 09.01.2008. 
werden können. Allen diesen Maßnahmen gemein ist die Tatsache, dass sie in erster Linie auf die Interessen der spanischen Wirtschaft ausgerichtet sind - dem zentralen Anliegen vieler Migranten nach einer dauerhaften Perspektive des Aufenthalts in Spanien werden sie nicht gerecht. ${ }^{21}$ Als Bestandteile einer kohärenten Migrationspolitik sind sie deshalb nur bedingt tauglich und laufen vielmehr Gefahr, die Fehlentwicklungen des nordwesteuropäischen Gastarbeitermodells zu reproduzieren.

Neben der Eindämmung der irregulären Zuwanderung und der Schaffung von regulären Zuwanderungsmöglichkeiten bildet die Integration der Migranten eine weitere Säule der sozialistischen Migrationspolitik. Lange Zeit spielte die Frage nach Perspektiven der Integration in der spanischen Diskussion eine untergeordnete Rolle. Erst in den letzten Jahren bemühten sich insbesondere die Autonomen Gemeinschaften um Integrationsmaßnahmen. Ein einheitliches Integrationskonzept ist dabei bislang nicht zu erkennen - die Bandbreite reicht von multikulturalistisch inspirierten Politiken in Katalonien bis zu assimilatorischen Ansätzen im konservativ regierten Valencia, wo Migranten - auf freiwilliger Basis - eine „Integrationsverpflichtung“ eingehen sollen, die unter anderem auch Kurse über Politik, Kultur und Sprache der Aufnahmegesellschaft umfasst. ${ }^{22}$ Die Regierung Zapatero brachte nun den Zentralstaat wieder als Akteur ins Spiel. 2007 wurde ein Integrationsplan für die Jahre bis 2010 beschlossen, der rund 2 Milliarden Euro für Maßnahmen insbesondere in den Bereichen Spracherwerb, Bildung und Arbeit vorsieht. ${ }^{23} 2008$ kündigte die sozialistische Regierung außerdem an, noch in der aktuellen Legislaturperiode ein Gesetz zum kommunalen Wahlrecht für Ausländer aus Staaten außerhalb der EU auf den Weg zu bringen.

Im Zuge der schweren Wirtschaftskrise, von der Spanien seit dem zweiten Halbjahr 2008 massiv betroffen war, begann sich auch der bislang dezidiert migrationsfreundliche Diskurs der sozialistischen Regierung zu wandeln. Das Ministerium für Arbeit und Immigration legte im Frühjahr 2009 ein - weitgehend wirkungsloses - Programm zur Rückkehrförderung von Migranten auf. Im Dezember 2009 verabschiedete das spanische Parlament eine Reform des Ausländergesetzes, die den Familiennachzug beschränkte und einige

Vgl. Enrique Bernales Ballesteros, Migración, Derechos Humanos, Migrantes Andinos a España. In: Secretaría General Iberoamericana. Encuentro Iberoamericano sobre Migración y desarrollo. Unidos por las migraciones. Madrid, 18 y 19 de julio de 2006. Madrid 2006, S. 237-245.

"Compromiso de integración." Comunitat Valenciana, Ley 15/2008, de 5 de diciembre, de integración de las personas inmigrantes en la Comunitat Valenciana, in: Boletín Oficial del Estado, Núm. 9, (2009), Título II, Del compromiso de integración, S. 3420. Zu Integrationspolitiken in Spanien allgemein vgl: Ruth Ferrero-Turrión, Gemma Pinyol-Jiménez, Immigration and the Construction of Public Philosophy(ies) of Integration in Spain, in: Elspeth Guild, Kees Groenendijk, Sergio Carrera (Hrsg.), Illiberal Liberal States, Immigration, Citizenship and Integration in the EU, Farnham, Burlington 2009, S. 337-356.

Baumer, (Fn. 8), S. 12. 
Maßnahmen gegen irreguläre Migration umfasste. ${ }^{24}$ Bemerkenswert ist allerdings, dass trotz der tiefen Krise und der extrem angespannten Haushaltslage des spanischen Staates nach wie vor ein Konsens darüber besteht, auch irregulären Migranten grundlegende soziale Rechte wie Gesundheitsversorgung oder Beschulung schulpflichtiger Kinder zu gewähren. Entsprechende Vorstöße einiger Bürgermeister, den Irregulären fortan die Registrierung im padrón municipal und damit den Zugang zu diesen öffentlichen Leistungen zu verwehren, wurden nicht nur von der regierenden PSOE und den kleineren Linksparteien, sondern auch von PP-Chef Mariano Rajoy zurückgewiesen. ${ }^{25}$

\section{Phasen der Migration aus Lateinamerika}

Lateinamerika war über lange Zeit hinweg das wichtigste Ziel spanischer Migranten. Insgesamt verließen zwischen 1850 und 1950 rund 3,5 Millionen Spanier ihre Heimat in Richtung Lateinamerika. Von der Remigration eines Teils dieser Auswanderer abgesehen, fand eine nennenswerte Wanderungsbewegung in entgegen gesetzter Richtung lange Zeit nicht statt. Das änderte sich erstmals mit der Errichtung der Militärdiktaturen in den Ländern des Cono Sur. Mit einem Mal gewann Spanien als Exilland an Bedeutung. In vielen Fällen mischten sich politische und ökonomische Motive, die Flucht vor politischer Verfolgung stand jedoch für diese Migrantengruppe eindeutig im Vordergrund. Nach den Demokratisierungsprozessen in den achtziger Jahren kehrte ein Teil dieser Migranten in ihre Ursprungsländer zurück, ein beträchtlicher Teil bleib jedoch in Spanien. ${ }^{26}$

Mit den ökonomischen und gesellschaftlichen Veränderungen Spaniens im Verlauf der achtziger Jahre, insbesondere der verstärkten Integration von Frauen in das Erwerbsleben, wuchs die Nachfrage nach ausländischen Arbeitskräften im häuslichen Dienstleistungssektor, wo die nun erwerbstätigen Spanierinnen ersetzt werden sollten. Diese Nachfragestruktur bildete zusammen mit den damals noch visumsfreien Einreisemöglichkeiten für Angehörige ehemaliger Kolonien den Rahmen für eine bedeutsame Zuwanderung von Migrantinnen aus der Karibik, insbesondere aus der Dominikanischen Republik.

Ein rassistischer Mord an einer Dominikanerin 1992 in einem Vorort von Madrid brachte die Präsenz dieser Migrantengruppe ins öffentliche Bewusstsein. Sowohl in der Wahrnehmung wie auch von ihrer Größe her stand die lateinamerikanische Migration jedoch lange Zeit im Schatten der Zuwanderung aus dem Maghreb und später aus den Ländern südlich der Sahara. Das änderte sich mit der Jahrtausendwende. Jetzt setzte ein regelrechter Boom der lateinamerikanischen Migration ein - zuerst aus Ecuador, Kolumbien und Peru, dann, in Folge der Krise, ab 2002 aus Argentinien und schließlich aus Boli-

Ley Orgánica 2/2009, de 11 de diciembre, de reforma de la Ley Orgánica 4/2000, de 11 de enero, sobre derechos y libertades de los extranjeros en España y su integración social, in: Boletín Oficial del Estado, Núm. 299 (2009), S. 104986 ff.

Vgl. $A B C, 22.10 .2010$.

26

Baumer, (Fn. 10), S. 171. 
vien. Die Einreise dieser Migranten erfolgte in den meisten Fällen regulär, zu Irregulären wurden sie erst nach Ablauf der Frist zu Ausreise.

Mit der Einführung der Visumspflicht für die wichtigsten Entsendeländer zwischen 2001 und 2003 hat sich der Migrationsstrom aus Lateinamerika etwas abgeschwächt. Insgesamt lässt sich jedoch sagen, dass sich die Arbeitsmigration aus Lateinamerika in den letzten Jahren auf einem sehr hohen Niveau stabilisiert hat. Die tiefe Krise, die Spaniens Wirtschaft seit dem ersten Halbjahr 2008 niederdrückt, hat zwar zu einem drastischen Rückgang der Zuwanderung geführt. ${ }^{27} \mathrm{Zu}$ einer nennenswerten Remigrationsbewegung ist es trotz der hohen Ausländerarbeitslosigkeit bislang nicht gekommen. Zumindest die lateinamerikanischen Migranten sind offensichtlich gekommen, um zu bleiben.

\section{Gegenwärtige Formen der Migration aus Lateinamerika}

Zum 1.1. 2009 verzeichneten die örtlichen Melderegister insgesamt 1.871.239 Migranten mit der Staatsangehörigkeit eines lateinamerikanischen Landes. Die größte nationale Gruppe stellen die Ecuadorianer dar, gefolgt von den Migranten aus Kolumbien, Bolivien, Argentinien und Peru. Nach Rumänen und Marokkanern bilden die Migranten aus Ecuador das größte nationale Kollektiv unter der ausländischen Bevölkerung Spaniens; Lateinamerika insgesamt ist die wichtigste Herkunftsregion für die Migration nach Spanien. ${ }^{28}$ Die Zuwanderung aus Lateinamerika nach Spanien entwickelt sich nach einem ähnlichen Muster wie andere Migrationströme: Am Anfang steht zumeist die reguläre Einreise als Tourist, drei Monate nach der Einreise bzw. nach Ablauf des Visums wird der Migrant zum Irregulären. Oder aber der irreguläre Aufenthalt schließt an eines der regulären, befristeten Arbeitsverhältnisse an, für die in den letzten Jahren verstärkt temporäre Aufenthalts- und Arbeitsgenehmigungen erteilt wurden. Die Arbeitssuche erfolgt dann meist im informellen Sektor, der bis in die Gegenwart, allen gegenläufigen Politiken zum Trotz, viele Beschäftigungschancen für Irreguläre bietet. Ein relativ stabiles Beschäftigungsverhältnis war in der Vergangenheit oftmals die Voraussetzung für eine Partizipation an Regularisierungsprozessen, die den bis dahin Irregulären erstmals eine befristete Aufenthalts- und Arbeitsgenehmigung einbrachten. Nach der ersten Erneuerung dieser Papiere nach einem Jahr setzte normalerweise eine Verfestigung des Aufenthaltsstatus ein. Am Ende dieses Prozesses stand vielfach die unbefristete Aufenthaltsgenehmigung oder die Einbürgerung. Seit 2005 hat es jedoch keine Regularisierungskampagne mehr gegeben - und auch in Zukunft soll keine massenhafte Regularisierung mehr stattfinden. Für die Irregulären, die zu spät kamen

Nach einem Bericht der OECD ging der Zustrom von Migranten nach Spanien 2008 im Vergleich zum Vorjahr um $43 \%$ zurück - so stark wie in keinem anderen OECD-Land. Die große Mehrheit der im Land lebenden Migranten scheint dagegen entschlossen, zu bleiben, obgleich diese Gruppe überproportional stark von der zunehmenden Arbeitslosigkeit betroffen ist. Programme der spanischen Regierung zur Rückkehrförderung stießen auf nur geringe Resonanz. Vgl. El País, 12.07.2010. 
oder aus anderen Gründen nicht an der letzten Regularisierung 2005 teilnehmen konnten, bedeutet dies, dass sie kaum Chancen auf eine Regularisierung ihrer Situation haben. ${ }^{29}$

Oftmals ist die Migration Teil einer „Familienstrategie ${ }^{* 30}$ und beginnt mit der Auswanderung eines Familienmitglieds. Gerade im Fall der Migranten aus den Ländern des Andenraums sind das in vielen Fällen Frauen, die erst nach einer Etablierungsphase ihre Partner, später auch Kinder und weitere Familienangehörige nachholen. Pilar Ponce hat für den Fall der ecuadorianischen Migration beschrieben, dass im Normalfall die Frau als erste migriert, nach einem Jahr der Mann und nach zwei weiteren Kinder und evtl. auch Großeltern nachkommen. ${ }^{31}$ Diese Migrationen finden meist noch unter Bedingungen der Irregularität statt. Bis vor wenigen Jahren konnte allerdings auf eine Regularisierung zu einem späteren Zeitpunkt gehofft werden - eine Perspektive, die sich für die Protagonisten der jüngsten Einwanderungswellen, insbesondere der aus Bolivien, wohl nicht mehr bietet.

Migranten lateinamerikanischer Herkunft sind in ihrem Verhältnis zur spanischen Aufnahmegesellschaft gegenüber anderen Migrantengruppen im Vorteil. Mit Ausnahme der relativ kleinen Gruppe der Brasilianer und einiger indígenas sind alle Migranten aus dieser Region spanischsprachig. Die gemeinsame Sprache erleichtert nicht nur die Integration in Arbeitswelt und Alltagsleben, sondern symbolisiert eine kulturelle Nähe, die zu einer erhöhten Akzeptanz in der Mehrheitsgesellschaft beiträgt - zumindest dort, wo das castellano noch als die gemeinsame Sprache akzeptiert ist. Eine vergleichbare, wenn auch längst nicht gleich bedeutende Rolle kommt der Religion zu. In Umfragen liegen Migranten aus Lateinamerika stets vor anderen Migrantenkollektiven, sowohl was die Häufigkeit des „täglichen Umgangs“ wie auch die „kulturelle Sympathie“، 32 angeht.

Jenseits dieses kulturellen Kapitals der Sprache und der Religion, das den meisten latinos zur Verfügung steht, unterscheiden sich die einzelnen Gruppen der lateinamerikanischen Migranten stark in ihrer jeweiligen sozialen und ökonomischen Situation. Neben Herkunft, Bildungshintergrund, Ausbildung, Dauer des Aufenthalts und anderen individuellen Faktoren ist es vor allem der Aufenthaltsstatus, der die soziale Situation determiniert. Der Umstand, ob ein Migrant die spanische Staatsbürgerschaft erlangt hat, über eine Aufenthaltsgenehmigung verfügt oder gar keine Papiere hat, sich also irregulär im Land befindet, entscheidet maßgeblich über Zugang zu und Qualität von Arbeit, Wohnung, staatlichen Leistungen, Partizipationsmöglichkeiten, sozialer Anerkennung etc. ${ }^{33}$ Und hier sind die Unterschiede zwischen den einzelnen Migrantenkollektiven aus Lateinamerika enorm.

Emilio José Gómez Ciriano, Andrés Tornos Cubillo, Colectivo Ioé, Ecuatorianos en España. Una aproximación sociológica, Madrid 2007, S. 92-93.

"Estrategia familiar." Ponce, (Fn. 4), S. 99.

31

Ebd., S. 101.

32

"Trato cotidiano", "simpatía cultural." Izquierdo Escribano et al., (Fn. 2), S. 19.

Javier de Lucas, Igualdad jurídica e inclusión política: por el empowerment de los migrantes, in: Secretaría General Iberoamericana, (Fn. 21), S. 248. 
Ingesamt stellt die irreguläre Migration nach wie vor die wohl größte Herausforderung für die Migrationspolitik der spanischen Regierung dar. Trotz der bislang umfassendsten Regularisierungskampagne im Jahr 2005, der in den letzten Jahren erweiterten Möglichkeiten der regulären Zuwanderung und dem verschärften Vorgehen gegen irreguläre Migranten geht die Zahl der sin papeles nur langsam zurück. Zum Jahreswechsel 2008/ 2009 betrug die Differenz zwischen der im padrón municipal registrierten Ausländern und der Zahl der Ausländer mit Aufenthaltsgenehmigungen ${ }^{34}$ 1.175.172, das entspricht einem Anteil von rund 20,80\% irregulärer Migranten an der gesamten ausländischen Wohnbevölkerung. 2007 waren es noch 1.497 .746 (33,14\%). Der Anteil der sin papeles an den Migranten aus Lateinamerika ist dabei überproportional hoch: 2007 betrug er 34,15\%, im Jahre 2008 32,04\%. 2009 verfügten noch immer 537.353 lateinamerikanische Migranten über keine Aufenthaltsgenehmigung. Das entspricht einem Anteil von 28,72\%. Dieser hohe Anteil verteilt sich sehr ungleich auf die verschiedenen lateinamerikanischen Nationalitäten, die auch bei den übrigen aufenthaltsrechtlichen Varianten (Staatsangehörigkeit oder Aufenthaltsgenehmigung) deutliche Unterschiede aufweisen. ${ }^{35}$

Die beste Voraussetzung für soziale und ökonomische Integration von Migranten stellt auch in Spanien die Einbürgerung dar. Wie weiter unten noch ausgeführt werden wird, sind lateinamerikanische Migranten im Allgemeinen und direkte Nachfahren spanischer Auswanderer im Besonderen bei der Erlangung der Staatsbürgerschaft bevorzugt. Dementsprechend viele der in Argentinien geborenen Migranten (33,79\%) sind spanische Staatsbürger, einen Status, den Angehörige der indigen geprägten Andenstaaten seltener erreichen. 25,39 \% der Peruaner, 17,41 \% der Kolumbianer, 14,57 \% der Ecuadorianer und lediglich $2,58 \%$ der Bolivianer haben die spanische Staatsbürgerschaft erlangt. ${ }^{36}$

Ganz anders stellt sich die Situation bei der Gruppe der lateinamerikanischen Migranten mit einem regulären Aufenthaltsstatus dar. Hier sind es die Migrantenkollektive, die vor (Dominikaner) oder zu Beginn des großen Booms (Ecuadorianer, Peruaner, Kolumbianer)

Der Stichtag für die Zahlen des padrón municipal ist der 1.1.2009, der für die Angaben über Aufenthaltsgenehmigungen für Ausländer im Anuario Estadístico 2008 der 31.12. 2008. Vgl. Instituto Nacional de Estadística, (Fn. 1); Ministerio de Trabajo e Inmigración, Secretaria de Estado de Inmigración y Emigración, Anuario Estadístico del año 2008, http://extranjeros. mtin.es/es/InformacionEstadistica/Anuarios/Anuario2008.html (27.07.2010); eigene Berechnungen. Alle folgenden Angaben beziehen sich auf das jeweilige Jahr des padrón municipal und die Angaben zum 31.12. des Vorjahrs aus der entsprechenden Ausgabe des Anuario Estadístico und auf eigene Berechnungen. Die Zahlen im padrón municipal gelten als leicht überhöht, da beispielsweise Wegzüge nicht registriert werden. Umgekehrt finden sich Aufenthaltsgenehmigungen, die sich gerade im Verlängerungsprozess befinden, nicht in der Statistik des Ministerio de Trabajo e Inmigración wieder. Darüber hinaus melden sich nicht alle Irregulären im padrón an. Trotz dieser Probleme führt diese Methode der Berechnung, auch nach Ansicht der meisten spanischen Migrationsforscher, zu einer relativ präzisen Annäherung an die tatsächliche Zahl der irregulären Migranten. Vgl. Carvajal Gómez, (Fn. 18), S. 88.

Instituto Nacional de Estadística, (Fn. 1); eigene Berechnungen.

Alle Angaben zum 01.01.2009. Vgl. Instituto Nacional de Estadística, (Fn. 1). 
nach Spanien kamen, die ihre aufenthaltsrechtliche Situation, oftmals unter Ausnutzung der verschiedenen Regularisierungen, inzwischen in großer Mehrheit konsolidieren konnten. Nahezu alle Migranten aus Ecuador ${ }^{37}$, 92,75 \% der Kolumbianer, 93,15\% der Peruaner und 93,35\% der Zuwanderer aus der Dominikanischen Republik verfügen über eine Aufenthaltsgenehmigung. Den später gekommen Migranten bleib der Weg in den in den regulären Aufenthalt mehrheitlich verwehrt. Nur 38,23\% der Bolivianer verfügen über eine Aufenthaltsgenehmigung, fast zwei Drittel der Angehörigen dieses Kollektivs sind irregulär im Land. Unter den argentinischen Migranten verfügen 49,74 \% über eine Aufenthaltserlaubnis. Die Gruppe der argentinischen Migranten zeichnet sich also durch eine hohe Einbürgerungsrate einerseits und einen hohen Anteil an Irregulären anderseits aus. Bei der argentinischen Migration nach Spanien handelt es sich mehrheitlich um relativ gut ausgebildete, junge Angehörige der urbanen Mittelschichten, die das Land massenhaft in den Jahren 2002 und 2003 verließen. ${ }^{38}$ Für die Mehrheit war die Migration eine direkte Reaktion auf die Folgen der Krise und stellte nicht notwendigerweise eine Entscheidung für eine längerfristige Auswanderung dar. Der, zumindest anfangs, temporäre Charakter der Migration und die relativ problemlose Integration in den spanischen Arbeitsmarkt dürften wohl mit dafür verantwortlich sein, dass für viele argentinische Migranten die Regularisierung ihres Aufenthalts keine Priorität hatte.

In der prekärsten Situation befindet sich ohne Zweifel das Kollektiv der bolivianischen Migranten. Der größte Teil der rund 230.000 registrierten Bolivianer kam erst in den letzten Jahren nach Spanien. Bis 2003 hatten sich lediglich 28.128 im padrón municipal angemeldet, 2005 waren es erst 96.367. Die wenigsten Bolivianer hatten also die Möglichkeit, von der letzten großen Regularisierung $2005 \mathrm{zu}$ profitieren. 2009 befanden sich $61,77 \%$ der Migranten aus Bolivien irregulär im Land. Sie haben kaum eine Chance, ihre Situation zu regularisieren. Als mehrheitlich gering qualifizierte Arbeiter sind sie besonders von den Folgen der Wirtschaftskrise, insbesondere in der Bauwirtschaft, betroffen. Der Verlust der Arbeit stellt für die Angehörigen dieser Migrantengruppe nicht nur ein existentielles Problem des Überlebens ohne ökonomische Ressourcen dar, sondern macht auch die letzte Hoffnung zunichte, über ein stabiles, wenn auch informelles, Arbeitsverhältnis in der Zukunft von einem weiteren Regularisierungsprozess oder einer individuellen Regularisie-

Tatsächlich sind in der Statistik des Ministeriums für Arbeit und Immigration zum Stichtag 31.12.2008 mehr ecuadorianische Migranten mit Aufenthaltsgenehmigung verzeichnet als im padrón municipal vom 1.1.2009 registriert sind, so dass sich rechnerisch ein Prozentsatz von 102,98 ergibt, was auf die weiter oben schon angemerkte Ungenauigkeit dieser beiden Statistiken zurückzuführen ist. Ein Jahr zuvor verfügten 96,01 \% der gemeldeten Ecuadorianer über eine Aufenthaltsgenehmigung. Es ist also davon auszugehen, dass zwischenzeitlich nahezu alle Migranten aus diesem Land ihre aufenthaltsrechtliche Situation regularisieren konnten. Alle Angaben: Ministerio de Trabajo e Inmigración, Secretaria de Estado de Inmigración y Emigración, (Fn. 34); Instituto Nacional de Estadística, (Fn. 1); eigene Berechnungen.

Alejandro Goldberg, Tú, sudaca. Las dimensiones histórico-geográficas, sociopolíticas y culturales alrededor del significado de ser inmigrante (y argentino) en España, Buenos Aires 2007, S. 40. 
rung aufgrund von „Verwurzelung“ profitieren zu können. Bolivianische Migranten sind also vom Syndrom der Konsequenzen von Irregularität in hohem Maße betroffen, das sich am besten mit dem Begriff der „Verletzlichkeit ““泩 ${ }^{39}$ eschreiben lässt.

Betrachtet man im Kontrast die Gruppe der ecuadorianischen Migranten, wird besonders deutlich, welche Konsequenzen der Zeitpunkt der Migration für die Integrationsperspektiven des jeweiligen Kollektivs hatte. Die Push-Faktoren waren für die gesamte andinisch geprägte Migration die gleichen: Armut, politische und gesellschaftliche Instabilität, Unsicherheit, Perspektivlosigkeit. ${ }^{40}$ Vergleichbar sind auch die sozialstrukturelle Zusammensetzung der Migrantenkollektive - mehrheitlich gering qualifiziert, ein hoher Anteil an indigenen Bevölkerungsgruppen, große Bedeutung der weiblichen Migration - genauso wie der Prozess der Integration in den Arbeitsmarkt. Trotzdem befindet sich die Mehrheit der ecuadorianischen Migranten in einer grundlegend verschiedenen Situation. ${ }^{41}$ Fast 15 Prozent der Ecuadorianer in Spanien verfügen inzwischen über die spanische Staatsbürgerschaft. Fast alle übrigen Migranten aus Ecuador haben einen stabilen Aufenthaltsstatus zumeist mit Aufenthaltsgenehmigungen von mehr als einem Jahr. Sie kamen mehrheitlich in den Jahren 2000 bis 2001 nach Spanien und konnten von den jüngsten Regularisierungsprozessen profitieren. Und nur eine kleine Minderheit von Ecuadorianern befindet sich in der Irregularität - entweder, weil sie auch die letzte Regularisierung verpasst haben, oder weil sie aufgrund von Arbeitslosigkeit ihre Aufenthaltserlaubnis verloren haben. ${ }^{42}$ Diese prekären Bedingungen, von denen inzwischen nur noch eine Minderheit der Ecuadorianer betroffen ist, stellen dagegen für die große Mehrheit der bolivianischen Migranten nach wie vor den Regelfall dar. Nicht von ungefähr protestierte Boliviens Präsident Evo Morales besonders scharf gegen die neue Rückführungsrichtlinie ${ }^{43}$, die Ministerrat und Parlament der EU im Frühsommer 2008 beschlossen haben. Ein härteres Vorgehen gegen irreguläre Migranten in Spanien und anderen Ländern der EU würde seine Landsleute in besonderem Maße betreffen.

Angesichts der tiefen Konjunkturkrise der spanischen Wirtschaft seit 2008 bleibt allerdings abzuwarten, wie sich die Situation der Migranten mit einem regulären Aufenthaltsstatus entwickelt. Befristete Aufenthaltsgenehmigungen sind zumeist an ein Beschäftigungsverhältnis gebunden - dauerhafte Arbeitslosigkeit kann also auch zu einer Gefährdung des Aufenthaltsstatus führen.

Die Integration der Migranten aus Lateinamerika in den spanischen Arbeitsmarkt weist einige Charakteristika auf, die sie von anderen Migrantengruppen unterscheiden. Im Jahr

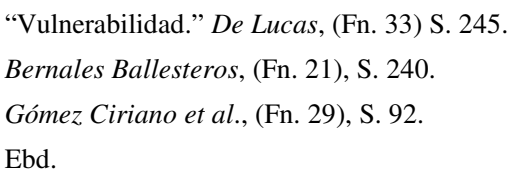

Morales bezeichnete das Regelwerk, das unter anderem eine Abschiebehaft für Irreguläre von bis zu 18 Monaten vorsieht, als eine „Richtlinie der Schande“ (,directiva de la vergüenza“). Vgl. http://news.bbc.co.uk/hi/spanish/international/newsid_7445000/7445052.stm (28.07.2010). 
$2008^{44}$ waren $74,67 \%$ der sozialversicherungspflichtig Beschäftigten im regulären Sozialversicherungssystem (Régimen General de la Seguridad Social) angemeldet. 5,29\% fielen unter das System für den Agrarbereich (Régimen Especial Agrario), 15,48 \% waren in dem System für Beschäftigte in privaten Haushalten registriert. (Régimen Especial de Empleados del Hogar) Zum Vergleich: Von den marokkanischen Beschäftigten waren im gleichen Jahr nur 57,82 \% im regulären, jedoch 34,48 \% im System für den Agrarbereich angemeldet. Sozialversicherungspflichtige Jobs in Haushalten machten bei dieser Gruppe nur $1,23 \%$ der Beschäftigungsverhältnisse aus. Betrachtet man die Verteilung in den Beschäftigungssektoren, die dem regulären Sozialversicherungssystem zugeordnet sind, werden die Unterschiede noch deutlicher. 75,45\% der Lateinamerikaner waren im Dienstleistungssektor beschäftigt, gegenüber 55,74 \% der marokkanischen Migranten. Diese waren dagegen zu 28,03 \% im Baugewerbe angemeldet, von den Lateinamerikanern waren es 15,19\%. Unter den einzelnen nationalen Kollektiven der lateinamerikanischen Migranten ist ebenfalls eine Spezifizierung zu konstatieren. Bolivianer und Ecuadorianer sind überproportional in der Landwirtschaft und in der Bauwirtschaft vertreten, während die große Mehrheit der Argentinier ihr Auskommen im Dienstleistungssektor gefunden hat.

\section{E. Los inmigrantes preferidos? Politik, Recht und öffentliche Meinung}

Migrationsprozesse sind stets das Ergebnis eines Zusammenspiels aus Push- und Pullfaktoren, also den Umständen, die im Herkunftsland und im Zielland auf die Migrationsentscheidung einwirken. $\mathrm{Zu}$ den Pull-Faktoren gehören konjunkturelle Faktoren, etwa die Nachfragesituation in bestimmten Segmenten des Arbeitsmarktes, genauso wie strukturelle Faktoren, beispielsweise einreise- und aufenthaltsrechtliche Bestimmungen. Aspekte wie gemeinsame Sprache, Religion, kulturelle Nähe etc. können ebenfalls eine wichtige Rolle spielen, insbesondere dann, wenn sie die Migrationspolitik des Aufnahmelandes beeinflussen. $^{45}$

Im Folgenden sollen nun diejenigen Faktoren untersucht werden, die Migration aus Lateinamerika nach Spanien gegenüber der aus anderen Regionen begünstigen. Daran wird sich die Diskussion der Frage anschließen, ob tatsächlich von einer Politik der Bevorzugung gesprochen werden kann und welche Rolle dabei das Bewusstsein einer gemeinsamen Geschichte oder Konzepte wie das der Hispanidad spielen.

Alle Angaben zum 31.12.2008. Vgl. Ministerio de Trabajo e Inmigración, Secretaria de Estado de Inmigración y Emigración, (Fn. 34). of Immigrants in European Societies. National Differences and Trends of Convergence, Stuttgart 2003, S. 9-14. 


\section{Rechtliche Rahmenbedingungen}

Lateinamerikanische Migranten haben gegenüber Zuwanderern aus anderen Regionen verschiedene rechtliche Vorteile:

- Abkommen über doppelte Staatsangehörigkeit: Im Rahmen der franquistischen Außenpolitik gegenüber Lateinamerika wurden solche Abkommen mit einer Reihe von Staaten geschlossen (mit Argentinien im Jahr 1969, Bolivien 1961, Chile 1958, Costa Rica 1964, Ecuador 1964, Guatemala 1961, Honduras 1966, Paraguay 1959, Peru 1959, Dominikanische Republik 1968, mit Kolumbien erst 1979). Die Entscheidung für eine Einbürgung in Spanien fällt für Migranten aus diesen Ländern leichter, da die alte Staatsbürgerschaft nicht aufgegeben werden muss. ${ }^{46}$

- Präferentieller Zugang zur spanischen Staatsbürgerschaft. Lateinamerikaner haben allgemein vereinfachten Zugang zur spanischen Staatsbürgerschaft. Kinder von Spaniern können die Staatsbürgerschaft sofort beantragen, das gleiche gilt seit 2003 auch für die direkten Nachfahren von lateinamerikanischen Bürgern, die früher die spanische Staatsbürgerschaft besessen haben (españoles de orígen). ${ }^{47}$ Nachfahren in der zweiten Generation können die Einbürgerung schon nach einem Jahr Aufenthalt in Spanien beantragen (españoles de residencia). Lateinamerikaner ohne spanische Vorfahren können nach zwei Jahren regulärem Aufenthalt in Spanien eingebürgert werden. Alle übrigen Migranten müssen vor der Einbürgerung mindestens zehn Jahre regulär in Spanien gelebt haben. Hier wird die „klare ethnische Grundausrichtung “48 des spanischen Staatsbürgerschaftsrechts deutlich. Auch die Statistik zeigt, dass Lateinamerikaner einen überproportionalen Anteil an der Zahl der Einbürgerungen haben. 2006 entfielen 82,52 \% aller Einbürgerungen auf dieses Kollektiv, für den Zeitraum 1991-2001, also noch vor dem Boom der lateinamerikanischen Zuwanderung, waren es $59 \%$ aller konzedierten Einbürgerungen. ${ }^{49}$

- Visumsfreiheit: Auch nach der Angleichung der Einreisebestimmungen an die der übrigen Schengen-Staat benötigten Angehörige lateinamerikanischer Staaten kein Visum, um nach Spanien einzureisen. Diese Regelung wurde erst in den Jahren 2001 bis 2003, also in der Hochphase des Migrationsbooms, für die wichtigsten Herkunftsländer (Kolumbien, Kuba, Ecuador, Peru und Dominikanische Republik) ausgesetzt.

Federación Iberoamericana de Ombudsman, I. Informe sobre Derechos humanos. Migraciones, Madrid 2003, S. 150.

Vgl. Andreu Domingo, Tras la retórica de la hispanidad: la migración latinoamericana en España entre la complementariedad y la exclusión, in: Papers de demografia, № 254 (2005).

Axel Kreienbrink, Länderprofil Spanien, Focus Migration Nr. 6, August 2008, S. 7. http://www. focus-migration.de/typo3_upload/groups/3/focus_Migration_Publikationen/Laenderprofile/LP_ 06_Spanien-08.pdf (23.07.2010).

Domingo, (Fn. 47), S. 4. 


\section{Politische Rahmenbedingungen}

Die Zuwanderungspolitik der spanischen Regierungen hat in den vergangenen Jahren versucht, dem Phänomen der massenhaften irregulären Migration über rein polizeiliche Maßnahmen hinaus mit zwei Ansätzen zu begegnen. Zum einen erhielten irreguläre Migranten in regelmäßigen Abständen die Gelegenheit, ihre aufenthaltsrechtliche Situation zu regularisieren. Zum anderen wurden die Möglichkeiten einer regulären Arbeitsmigration ausgeweitet. Von beiden Politiken konnten lateinamerikanische Migranten überproportional profitieren.

In den beiden großen Regularisierungsprozessen, die die konservative Regierung von José María Aznar in den Jahren 2000 und 2001 organisierte, wurden 58,7 \% der Antragssteller anerkannt - unter den Lateinamerikanern betrug diese Quote 76,7 \%. ${ }^{50}$ Auch von der bislang größten Maßnahme dieser Art, die von der sozialistischen Regierung 2005 unter der programmatischen Bezeichnung „Prozess der Normalisierung des Status ausländischer Arbeiter “51 durchgeführt wurde, konnten die irregulären Arbeitsmigranten aus Lateinamerika besonders profitieren.

Auch in der Frage des regulären Zugangs auf den spanischen Arbeitsmarkt befinden sich die Migranten aus Lateinamerika in einer relativ guten Position: 2001 beschloss die spanische Regierung das Programm GRECO ${ }^{52}$, das u.a. als Grundlage für eine Reihe von bilateralen Abkommen über Arbeitsmigration mit Ländern dienen sollte, die nach politischen, ökonomischen und kulturellen Motiven ausgewählt wurden. Die ersten Abkommen wurden 2001 mit Ecuador, Kolumbien und Marokko geschlossen (letzteres jedoch aufgrund der politischen Spannungen erst 2005 ratifiziert). Es folgten weitere Verträge mit der Dominikanischen Republik (2002), Polen, Rumänien und Bulgarien (2003). Mit der signifikanten Ausnahme des Abkommens mit Marokko, das auf dessen damaligen Status als wichtigstes Herkunftsland zurückgeht, war der Versuch der konservativen Regierung unverkennbar, mit diesen Abkommen Migrationsströme nach einem Konzept der „kulturellen Nähe“"53 zu leiten. Die direkte Wirksamkeit dieser Abkommen war begrenzt. Zwischen 2001 und 2005 wurden beispielsweise nur rund 2000 ecuadorianische Arbeitsmigranten im Rahmen des bilateralen Abkommens vermittelt. Im Rahmen des Abkommens

51.

"Proceso de normalización documental de trabajadores extranjeros." Ministerio de Trabajo y Asuntos Sociales, Secretaría de Estado de Inmigración y Emigración. Boletín estadístico de extranjería e inmigración, Número 7, Enero 2006, S. 3. http://extranjeros.mtin.es/es/ InformacionEstadistica/Boletines/Archivos/Boletin-Num-07-Web.pdf (27.07.2010)

"Programa Global de Regularización y Coordinación de Extranjería y de Inmigración.” Kreienbrink, (Fn. 15), S. 436-444.

53 "Proximidad cultural." Axel Kreienbrink, Inmigración e integración social de los inmigrantes en España entre consenso y enfrentamiento político, in: Walther L Bernecker / Günther Maihold (Hrsg.), España: del consenso a la polarización. Cambios en la democracia española, Madrid, Frankfurt 2007, S. 259. 
zwischen Kolumbien und Spanien erhielten im gleichen Zeitraum 16.000 Kolumbianer eine befristete Arbeitserlaubnis. ${ }^{54}$ Allerdings kommen auch die übrigen Instrumente zur Steuerung der Arbeitsmigration - jährliche Kontingente für bestimmte Beschäftigungen, Saisonarbeit - vornehmlich den Ländern zugute, mit denen ein solches bilaterales Abkommen besteht. $^{55}$

\section{III. Öffentliche Meinung}

Verschiedene Autoren ${ }^{56}$ haben darauf hingewiesen, dass Umfragen immer wieder die Präferenz eines großen Teils der spanischen Bevölkerung für Migranten aus Lateinamerika gegenüber Zuwanderern aus anderen Weltregionen ergeben haben: „Sie werden von den Unternehmern und den Politikern, von den Einwohner der Stadtviertel, den katholischen Organisationen und von den Lehrern in den Schulen bevorzugt. “57 Dabei stellen die gemeinsame Sprache und die in der kollektiven Vorstellung der Aufnahmegesellschaft weit verbreitete Idee der kulturellen Nähe die wichtigsten Faktoren für diese positive Wahrnehmung der lateinamerikanischen Migration dar. Gleichzeitig wurde eine verstärkte Zuwanderung aus Lateinamerika auch vielfach als eine Möglichkeit wahrgenommen, die Abhängigkeit von marokkanischer Arbeitskraft in bestimmten Wirtschaftszweigen zu verringern und damit letztendlich auch dem ,kulturellen Zusammenstoß mit einer anderen Religion“ ${ }^{58}$ aus dem Weg zu gehen. Die positive Bezugnahme auf die lateinamerikanische Migration und die Betonung der sprachlichen und kulturellen Nähe ging stets einher mit einem impliziten Ressentiment gegenüber Migranten aus anderen Regionen. ${ }^{59}$ Auf der politischen Ebene wurde diese gesellschaftliche Präferenz gegenüber lateinamerikanischen Migranten in einen Diskurs integriert, der „eine klare Bevorzugung für die Migranten aus dieser Region im Namen der historischen und sprachlichen Gemeinschaft, wie sie in der hispanidad verkörpert wird“"60 ${ }^{60}$ einschloss.

Der Verweis auf das Konzept der hispanidad findet sich im offiziellen Diskurs der Regierung normalerweise zwar nicht, gleichwohl wird das Thema der lateinamerikanischen Migration stets in den Kontext der Iberoamerikanischen Staatengemeinschaft gestellt, mithin also der demokratisch recycelten Version der hispanidad. Unter dem Titel „Vereint

Günther Müssig, Acuerdos y Programas de Migración Temporal, in: Secretaría General Iberoamericana, (Fn. 21), S. 370.

Marta Rodríguez Tarduchi, Acuerdos y Programas de Migración Temporal, in: ebd., S. 389.

Domingo, (Fn. 47), Izquierdo Escribano et al., (Fn. 2).

57 "Los prefieren los empresarios y los políticos, los vecinos del barrio, las organizaciones católicas y los maestros en los colegios.” Izquierdo Escribano et al., (Fn. 2), S. 3.

"Choque cultural con otra religión." Ebd.

Domingo, (Fn. 47), S. 2.

"Una clara preferencia por los migrantes procedentes de ese territorio en nombre de la comunidad histórica y lingüística que supone la hispanidad.” Ebd., S. 19. 
durch Migration: Iberoamerikanische Konferenz über Migration und Entwicklung “61 ${ }^{\text {fand }}$ im Sommer 2006 ein großes Symposium zu diesem Thema in Madrid statt. Ein Jahr zuvor hatte die Konferenz der iberoamerikanischen Staats- und Regierungschefs auf ihrem Gipfeltreffen in Salamanca dem iberoamerikanischen Generalsekretariat das Mandat für die Vorbereitung einer solchen Konferenz erteilt. Marta Rodríguez Tarduchi, zu diesem Zeitpunkt Generaldirektorin für Immigration im damaligen Ministerium für Arbeit und soziale Angelegenheiten $^{62}$, hat auf dieser Bezugsrahmen der spanischen Migrationspolitik gegenüber Lateinamerika umrissen: ${ }^{63}$

Diese historischen Überlegungen, die im Kontext der iberoamerikanischen Gemeinschaft angestellt werden, müssen an die Solidarität und die Gastfreundschaft vieler Länder Lateinamerikas erinnern, die vor noch nicht allzu langer Zeit viele Tausend wirtschaftliche und politische Migranten aus Spanien großzügig empfangen haben, die vor einer feindlichen Gegenwart flohen und in Amerika Unterstützung und Chancen vorfanden. Die notwendige Bestimmtheit der Politik und die Verpflichtung, die Migrationströme kontrolliert und geordneten zu verwalten, dürfen in Bezug auf die iberoamerikanische Gemeinschaft nicht dazu führen, dass wir diese gemeinsame Geschichte vergessen, die Bindungen zwischen beiden Küsten des Atlantiks geschaffen hat, welche die konkreten historischen Konjunkturen, die die einzelnen Gesellschaften durchlaufen, transzendieren.

Beide Elemente dieser Stellungnahme - die spanische Emigration nach Lateinamerika und die alles überwölbende gemeinsame Geschichte - bilden den Rahmen, in dem sich die spanische Migrationspolitik gegenüber Lateinamerika entwickelt hat. Die Lateinamerikanisierung der Migration nach Spanien hat hier ihre Wurzeln.

\section{F. Fazit}

Die Migration aus Lateinamerika gleicht in Form und Verlauf in vielerlei Hinsicht den Zuwanderungen aus anderen Regionen nach Spanien. Die Kombination von Push-Faktoren, die in den Herkunftsländern große Gruppen von Menschen zur Migration getrieben hat, ist in vielen Fällen eine ähnliche. Das gilt auch für eine Reihe von Pull-Faktoren, allen 22.

Inzwischen hat sich Denomination geändert: die neue Bezeichnung lautet „Ministerium für Arbeit und Immigration“ (Ministerio de Trabajo e Inmigración).

"Esta reflexión histórica, formulada en el contexto de la comunidad iberoamericana, no puede dejar de evocar la solidaridad y la hospitalidad de tantos países de América Latina que, no hace tantos años, recibieron con generosidad la inmigración económica y el exilio político de miles y miles de españoles que huían de una realidad hostil y dramática y encontraron en América solidaridad y oportunidades. El rigor en la determinación de las políticas y el imperativo de gestionar controlada y ordenadamente los flujos migratorios no pueden, cuando hablamos de la comunidad iberoamericana hacernos olvidar esta historia común que establece entre ambas orillas del Atlántico vínculos que trascienden las coyunturas históricas concretas por las que atraviesen unas y otras sociedades.” Rodríguez Tarduchi, (Fn. 55), S. 385. 
voran die lange Zeit ungebremste Nachfrage in bestimmten Segmenten des spanischen Arbeitsmarktes.

Die lateinamerikanische Migration wurde darüber hinaus durch rechtliche, politische und gesellschaftliche Rahmenbedingungen spezifisch gefördert, die den regelrechten Boom um die Jahrtausendwende erst ermöglichten. Strukturelle Faktoren wie günstige Einreiseund Aufenthaltsbestimmungen, aber auch die gemeinsame Sprache und die relativ hohe Akzeptanz in der Aufnahmegesellschaft erwiesen sich als mächtige Sogfaktoren, die innerhalb weniger Jahre zu einer massenhaften Zuwanderung aus Lateinamerika führten. Spätestens ab der Jahrtausendwende wurde diese Dynamik durch eine aktive Politik der Regierung noch verstärkt: Die Unterzeichnung von bilateralen Abkommen mit den wichtigsten Entsendeländern, die Änderung des Staatsbürgerschaftsrechts 2003 und die Bevorzugung von Lateinamerikanern in den verschiedenen Regularisierungsprozessen spricht dafür, dass die Migranten aus Lateinamerika tatsächlich als die ,preferidos del siglo XXI“ ${ }^{64}$ betrachtet wurden - wenn auch nicht als die einzigen, wie die ebenfalls geförderte Zuwanderung von Migranten aus Mittelosteuropa belegt. Dabei ist hervorzuheben, dass die Regierung mit ihrer Politik die Präferenz von großen Teilen der Gesellschaft für eine Zuwanderung aus Lateinamerika aufgegriffen und verstärkt hat. Für die klare Vorliebe, die die Spanier nach allen Umfragen den Migranten aus Lateinamerika gegenüber denen aus anderen Regionen entgegen bringen, dürfte das Bewusstsein der gemeinsamen Geschichte oder gar eine Wertschätzung der hispanidad nur eine untergeordnete Rolle spielen. Vielmehr sind es die gemeinsame Sprache und die kulturelle Nähe auf der einen und ein mehr oder minder bewusstes Ressentiment gegenüber den Marokkanern und noch allgemeiner gegenüber der erinnerungsmächtigen Figur des moro auf der anderen Seite, die diese Präferenz konstituieren.

Für die Lateinamerikaner bedeutet ihr kulturelles Kapital einen wesentlichen Vorteil bei der Integration in die Aufnahmegesellschaft. Dabei sollte aber nicht vergessen werden, dass die relativ reibungslose Absorption der Migranten in den spanischen Arbeitsmarkt die ungleich wichtigere Integrationsvoraussetzung war - sollte diese im Kontext der wirtschaftlichen Krise wegfallen, könnte sich auch das relative Wohlwollen der spanischen Gesellschaft gegenüber den latinos rasch verflüchtigen. Das Bewusstsein der gemeinsamen Geschichte mag historische Konjunkturen überdauern. Die durch Sprache und Kultur geförderte Akzeptanz der Aufnahmegesellschaft für eine bestimmte Migrantengemeinde könnte dagegen empfindlich auf wirtschaftliche Konjunkturen reagieren. 